\title{
ONE-POINT EXTENSIONS AND LOCAL TOPOLOGICAL PROPERTIES
}

\author{
M. R. KOUSHESH \\ (Received 16 May 2012; accepted 2 June 2012; first published online 2 August 2012)
}

\begin{abstract}
A space $Y$ is called an extension of a space $X$ if $Y$ contains $X$ as a dense subspace. An extension $Y$ of $X$ is called a one-point extension of $X$ if $Y \backslash X$ is a singleton. P. Alexandroff proved that any locally compact non-compact Hausdorff space $X$ has a one-point compact Hausdorff extension, called the onepoint compactification of $X$. Motivated by this, Mrówka and Tsai ['On local topological properties. II', Bull. Acad. Polon. Sci. Sér. Sci. Math. Astronom. Phys. 19 (1971), 1035-1040] posed the following more general question: For what pairs of topological properties $\mathscr{P}$ and $\mathscr{Q}$ does a locally- $\mathscr{P}$ space $X$ having $\mathscr{Q}$ possess a one-point extension having both $\mathscr{P}$ and $\mathscr{Q}$ ? Here, we provide an answer to this old question.
\end{abstract}

2010 Mathematics subject classification: primary 54D35.

Keywords and phrases: Stone-Čech compactification, one-point extension, one-point compactification, Mrówka's condition $W$.

\section{Introduction}

Let $\mathscr{P}$ be a topological property.

- $\quad \mathscr{P}$ is closed hereditary if any closed subspace of a space with $\mathscr{P}$, also has $\mathscr{P}$.

- $\quad \mathscr{P}$ is preserved under finite closed sums if any space which is expressible as a finite union of closed subspaces each having $\mathscr{P}$, also has $\mathscr{P}$.

- $\quad \mathscr{P}$ satisfies Mrówka's condition $(W)$ if it satisfies the following: if $X$ is a completely regular space in which there exists a point $p$ with an open base $\mathscr{B}$ for $X$ at $p$ such that $X \backslash B$ has $\mathscr{P}$ for any $B \in \mathscr{B}$, then $X$ has $\mathscr{P}$. (See [10].)

REMARK 1.1. If $\mathscr{P}$ is a topological property which is closed hereditary and productive then Mrówka's condition $(W)$ is equivalent to the following condition: if a completely regular space $X$ is the union of a compact space and a space with $\mathscr{P}$, then $X$ has $\mathscr{P}$. (See [8].)

Let $X$ be a space and let $\mathscr{P}$ be a topological property. The space $X$ is called locally$\mathscr{P}$ if each of its points has a neighbourhood in $X$ with $\mathscr{P}$. Note that if $X$ is regular

This research was in part supported by a grant from IPM (No. 90030052).

(C) 2012 Australian Mathematical Publishing Association Inc. 0004-9727/2012 \$16.00 
and $\mathscr{P}$ is closed hereditary, then $X$ is locally- $\mathscr{P}$ if and only if each $x \in X$ has an open neighbourhood $U$ in $X$ such that $\mathrm{cl}_{X} U$ has $\mathscr{P}$.

Let $X$ and $E$ be Hausdorff spaces. The space $X$ is said to be $E$-completely regular if $X$ is homeomorphic to a subspace of a product $E^{\alpha}$ for some cardinal $\alpha$. (See $[3,11]$.) In [12] (see also [14]) the authors proved that for a topological property $\mathscr{P}$ which is regular-closed hereditary and preserved under finite closed sums, and satisfies Mrówka's condition $(W)$, every $E$-completely regular (where $E$ is regular and subject to some restrictions) locally- $\mathscr{P}$ space has a one-point $E$-completely regular extension having $\mathscr{P}$. (See [9] for related results.) The authors then posed the following more general question: For what pairs of topological properties $\mathscr{P}$ and $\mathscr{Q}$ is it true that every locally- $\mathscr{P}$ space having $\mathscr{Q}$ possesses a one-point extension having both $\mathscr{P}$ and $\mathscr{Q}$ ? Indeed, the systematic study of questions of this sort dates back to earlier times when P. Alexandroff proved that every locally compact non-compact Hausdorff space has a one-point compact Hausdorff extension (thus answering the question in the case where $\mathscr{P}$ is compactness and $\mathscr{Q}$ is the Hausdorff property). Since then the question has been considered by various authors for specific choices of topological properties $\mathscr{P}$ and $\mathscr{Q}$. In this note we provide an answer to the above old question of Mrówka and Tsai. (See also [7, Theorem 4.1] for a related result.) The results of this note modify and simplify those we have proved in the final chapter of [4].

We now review some notation and terminologies. For undefined terms and notation we refer to [2].

Let $X$ be a space. If $f: X \rightarrow \mathbb{R}$ is continuous, write $\operatorname{Coz}(f)=X \backslash f^{-1}(0)$. Let

$$
\operatorname{Coz}(X)=\{\operatorname{Coz}(f): f: X \rightarrow \mathbb{R} \text { is continuous }\} .
$$

Let $X$ be a completely regular space. The Stone-Čech compactification $\beta X$ of $X$ is the compactification of $X$ characterised among all compactifications of $X$ by the following property: every continuous $f: X \rightarrow[0,1]$ is continuously extendable over $\beta X$; denote by $f_{\beta}$ this continuous extension of $f$.

\section{One-point $\mathscr{P}$ - $\mathscr{Q}$-extensions of locally- $\mathscr{P}$ non- $\mathscr{P} \mathscr{Q}$-spaces}

The following subspace of $\beta X$, introduced in [4] (also in [5]), plays a crucial role.

Definition 2.1. For a completely regular space $X$ and a topological property $\mathscr{P}$, let

$$
\lambda_{\mathscr{P}} X=\bigcup\left\{\operatorname{int}_{\beta X} \mathrm{cl}_{\beta X} C: C \in \operatorname{Coz}(X) \text { and } \operatorname{cl}_{X} C \text { has } \mathscr{P}\right\} .
$$

ReMark 2.2. If $\mathscr{P}$ is pseudocompactness then

$$
\lambda_{\mathscr{P}} X=\operatorname{int}_{\beta X} v X
$$

where $v X$ is the Hewitt realcompactification of $X$. (See $[5,6]$.)

If $X$ is a space and $D$ is a dense subspace of $X$, then $\operatorname{cl}_{X} U=\operatorname{cl}_{X}(U \cap D)$ for every open subspace $U$ of $X$. We have the following simple observation. 
Lemma 2.3. Let $X$ be a completely regular space and let $f: X \rightarrow[0,1]$ be continuous. If $0<r<1$ then

$$
f_{\beta}^{-1}([0, r)) \subseteq \operatorname{int}_{\beta X} \mathrm{cl}_{\beta X} f^{-1}([0, r)) .
$$

Proof. Note that

$$
f_{\beta}^{-1}([0, r)) \subseteq \operatorname{cl}_{\beta X} f_{\beta}^{-1}([0, r))=\operatorname{cl}_{\beta X}\left(X \cap f_{\beta}^{-1}([0, r))\right)=\operatorname{cl}_{\beta X} f^{-1}([0, r)) .
$$

This concludes the proof.

Lemma 2.4. Let $X$ be a completely regular locally- $\mathscr{P}$ space, where $\mathscr{P}$ is a closed hereditary topological property. Then $X \subseteq \lambda_{\mathscr{P}} X$.

Proof. Let $x \in X$ and let $U$ be an open neighbourhood of $x$ in $X$ whose closure $\operatorname{cl}_{X} U$ has $\mathscr{P}$. Let $f: X \rightarrow[0,1]$ be continuous with $f(x)=0$ and $f \mid(X \backslash U) \equiv 1$. Let $C=f^{-1}([0,1 / 2)) \in \operatorname{Coz}(X)$. Then $C \subseteq U$ and thus $\operatorname{cl}_{X} C$ has $\mathscr{P}$, as it is closed in $\operatorname{cl}_{X} U$. Therefore $\operatorname{int}_{\beta X} \mathrm{cl}_{\beta X} C \subseteq \lambda_{\mathscr{P}} X$. But then $x \in \lambda_{\mathscr{P}} X$, as $x \in f_{\beta}^{-1}([0,1 / 2))$ and $f_{\beta}^{-1}([0,1 / 2)) \subseteq \operatorname{int}_{\beta X} \operatorname{cl}_{\beta X} C$ by Lemma 2.3.

THEOREM 2.5. Let $\mathscr{P}$ be a closed hereditary topological property preserved under finite closed sums and satisfying Mrówka's condition $(W)$. Let $\mathscr{Q}$ be a closed hereditary topological property satisfying Mrówka's condition $(W)$ and implying complete regularity. If $X$ is a locally- $\mathscr{P}$ non- $\mathscr{P}$ space having $\mathscr{Q}$ then $X$ has a onepoint extension having both $\mathscr{P}$ and $\mathscr{Q}$.

Proof. Let $X$ be a locally- $\mathscr{P}$ non- $\mathscr{P}$ space having $\mathscr{Q}$. Note that $\lambda_{\mathscr{P}} X \neq \beta X$; as otherwise, by compactness and the definition of $\lambda_{\mathscr{P}} X$,

$$
\beta X=\operatorname{int}_{\beta X} \operatorname{cl}_{\beta X} C_{1} \cup \cdots \cup \operatorname{int}_{\beta X} \mathrm{cl}_{\beta X} C_{n}
$$

where $C_{1}, \ldots, C_{n} \in \operatorname{Coz}(X)$ and each $\operatorname{cl}_{X} C_{1}, \ldots, \mathrm{cl}_{X} C_{n}$ has $\mathscr{P}$. Taking the intersection of both sides of (2.1) with $X$,

$$
X=\operatorname{cl}_{X} C_{1} \cup \cdots \cup \mathrm{cl}_{X} C_{n} .
$$

This implies that $X$ has $\mathscr{P}$, as it is the finite union of closed subspaces each having $\mathscr{P}$. This is a contradiction. Note that $\lambda_{\mathscr{P}} X$ is open in $\beta X$ by its definition, and $X \subseteq \lambda_{\mathscr{P}} X$ by Lemma 2.4 , as $X$ is locally- $\mathscr{P}$. Let $T$ be the quotient space of $\beta X$ obtained by contracting the nonempty set $\beta X \backslash \lambda_{\mathscr{P}} X$ to a point $p$ and denote by $q: \beta X \rightarrow T$ its quotient mapping. Note that $T$ is Hausdorff, as $\beta X \backslash \lambda_{\mathscr{P}} X$ is closed in the normal space $\beta X$. Since $T$ is also compact, as it is the continuous image of $\beta X$, it is then completely regular. Also, note that $T$ contains $X$ as a dense subspace. Consider the subspace $Y=X \cup\{p\}$ of $T$. Then $Y$ is a completely regular one-point extension of $X$. We need to show that $Y$ has both $\mathscr{P}$ and $\mathscr{Q}$. To show this, since $\mathscr{P}$ and $\mathscr{Q}$ both satisfy Mrówka's condition $(W)$ it suffices to show that $Y \backslash V$ has $\mathscr{P}$ and $\mathscr{Q}$ for every open neighbourhood $V$ of $p$ in $Y$. Let $V$ be an open neighbourhood of $p$ in $Y$. Let $V^{\prime}$ be open in $T$ such that $V=Y \cap V^{\prime}$. Note that

$$
Y \backslash V=X \cap\left(T \backslash V^{\prime}\right)=X \cap q^{-1}\left(T \backslash V^{\prime}\right) .
$$


Since $p \in V^{\prime}$,

$$
q^{-1}\left(T \backslash V^{\prime}\right) \cap\left(\beta X \backslash \lambda_{\mathscr{P}} X\right)=q^{-1}\left(T \backslash V^{\prime}\right) \cap q^{-1}(p)=\emptyset
$$

and thus $q^{-1}\left(T \backslash V^{\prime}\right) \subseteq \lambda_{\mathscr{P}} X$. Since $q^{-1}\left(T \backslash V^{\prime}\right)$ is compact, as it is closed in $\beta X$,

$$
q^{-1}\left(T \backslash V^{\prime}\right) \subseteq \operatorname{int}_{\beta X} \operatorname{cl}_{\beta X} D_{1} \cup \cdots \cup \operatorname{int}_{\beta X} \operatorname{cl}_{\beta X} D_{m}
$$

for some $D_{1}, \ldots, D_{m} \in \operatorname{Coz}(X)$ such that each $\operatorname{cl}_{X} D_{1}, \ldots, \operatorname{cl}_{X} D_{m}$ has $\mathscr{P}$. Taking the intersection of both sides of (2.2) with $X$,

$$
Y \backslash V \subseteq \mathrm{cl}_{X} D_{1} \cup \cdots \cup \mathrm{cl}_{X} D_{m}=H .
$$

But $H$ has $\mathscr{P}$, as it is the finite union of closed subspaces each having $\mathscr{P}$. Therefore $Y \backslash V$ has $\mathscr{P}$, as it is closed in $H$. That $Y \backslash V$ has $\mathscr{Q}$ follows, as $Y \backslash V$ is closed in $X$ and $X$ has $\mathscr{Q}$.

ExAmple 2.6. The list of topological properties satisfying the assumption of Theorem 2.5 is quite long and includes almost all important covering properties (that is, topological properties described in terms of the existence of certain kinds of open subcovers or refinements of a given open cover of a certain type), among them compactness, countable compactness (more generally, $[\theta, \kappa]$-compactness), the Lindelöf property (more generally, the $\mu$-Lindelöf property), paracompactness, metacompactness, countable paracompactness, subparacompactness, submetacompactness (or $\theta$-refinability) and the $\sigma$-para-Lindelöf property. (See $[1,13]$ for definitions. For the proof that these all satisfy Mrówka's condition $(W)$, see [4]. That these topological properties are closed hereditary and preserved under finite closed sums follows from [1, Theorems 7.1, 7.3 and 7.4].)

\section{Acknowledgement}

The author wishes to thank the anonymous referee for reading the manuscript and the prompt report given within two weeks.

\section{References}

[1] D. K. Burke, 'Covering properties', in: Handbook of Set-Theoretic Topology, (eds. K. Kunen and J. E. Vaughan) (Elsevier, Amsterdam, 1984), pp. 347-422.

[2] R. Engelking, General Topology, 2nd edn (Heldermann, Berlin, 1989).

[3] R. Engelking and S. Mrówka, 'On E-compact spaces', Bull. Acad. Polon. Sci. Sér. Sci. Math. Astr. Phys. 6 (1958), 429-436.

[4] M. R. Koushesh, 'Compactification-like extensions', Dissertationes Math. (Rozprawy Mat.) 476 (2011), 88pp.

[5] M. R. Koushesh, 'The partially ordered set of one-point extensions', Topology Appl. 158 (2011), 509-532.

[6] M. R. Koushesh, 'A pseudocompactification', Topology Appl. 158 (2011), 2191-2197.

[7] J. Mack, M. Rayburn and R. G. Woods, 'Local topological properties and one-point extensions', Canad. J. Math. 24 (1972), 338-348. 
[8] J. Mack, M. Rayburn and R. G. Woods, 'Lattices of topological extensions', Trans. Amer. Math. Soc. 189 (1974), 163-174.

[9] F. L. Marin, 'A note on E-compact spaces', Fund. Math. 76 (1972), 195-206.

[10] S. Mrówka, 'On local topological properties', Bull. Acad. Polon. Sci. 5 (1957), 951-956.

[11] S. Mrówka, 'Further results on E-compact spaces. I', Acta Math. 120 (1968), 161-185.

[12] S. Mrówka and J. H. Tsai, 'On local topological properties. II', Bull. Acad. Polon. Sci. Sér. Sci. Math. Astronom. Phys. 19 (1971), 1035-1040.

[13] R. M. Stephenson Jr, 'Initially $\kappa$-compact and related spaces', in: Handbook of Set-Theoretic Topology, (eds. K. Kunen and J. E. Vaughan) (Elsevier, Amsterdam, 1984), pp. 603-632.

[14] J. H. Tsai, 'More on local topological properties', Bull. Acad. Polon. Sci. Sér. Sci. Math. Astronom. Phys. 22 (1974), 49-51.

M. R. KOUSHESH, Department of Mathematical Sciences, Isfahan University of Technology, Isfahan 84156-83111, Iran

and

School of Mathematics,

Institute for Research in Fundamental Sciences (IPM),

P.O. Box: 19395-5746, Tehran, Iran

e-mail: koushesh@cc.iut.ac.ir 